\title{
On the Fixed-Point Set and Commutator Subgroup of an Automorphism of a Group of Finite Rank
}

\author{
B. A. F. WEHRFRITZ
}

ABSTRACT - Let $\phi$ be an automorphism of a group $G$. For $G$ polycyclic, Endimioni and Moravec in [1] discuss the relationship between the fixed-point set $C_{G}(\phi)$ and the commutator subgroup $[G, \phi]$ of $\phi$ in $G$. Here we extend these results to soluble groups satisfying various rank restrictions.

Suppose $\phi$ is an automorphism of the polycyclic group $G$. Endimioni and Moravec in [1] prove the following.

i) If $C_{G}(\phi)$ is finite and $\phi$ has order 2 , then $[\mathrm{G}, \phi]^{\prime}$ is finite.

ii) If $C_{G}(\phi)$ is finite, then so is $G /[G, \phi]$.

iii) If $|\phi|$ is finite, then so is the index $\left(G:[G, \phi] . C_{G}(\phi)\right)$.

Of course $C_{G}(\phi)$ denotes the set of fixed points of $\phi$ in $G,[G, \phi]=$ $\left\langle g^{-1} . g \phi: g \in G\right\rangle$ and $[\mathrm{G}, \phi]$ is a $\phi$-invariant normal subgroup of $G$. Here we generalize these results to groups with some sort of rank restriction. Generally our groups at least have finite Hirsch number and satisfy something weaker than the FAR condition. We must start by defining these terms.

If a group $G$ has a series of finite length each factor of which is either locally finite or infinite cyclic, then the number of infinite cyclic factors in such a series is an invariant of $G$, which we call the Hirsch number of $G$ (it is also sometimes called the torsion-free rank of $G$ ). This is the weakest of the rank restrictions considered here. The structure of groups with finite Hirsch number is discussed in many places (e.g. see [3]). From our point of view the most convenient description of the structure of such a group is

(*) Indirizzo dell'A.: School of Mathematical Sciences, Queen Mary University of London, Mile End Road, London E1 4NS, England.

E-mail: b.a.f.wehrfritz@qmul.ac.uk 
given by Lemma 4 of [6]. A group satisfies min- $p$ for the prime $p$ if its $p$ subgroups satisfy the minimal condition on subgroups. An FAR group (that is, a finite-abelian-ranks group, also called an $\boldsymbol{S}_{\text {o group) is a soluble }}$ group with finite Hirsch number that satisfies min- $p$ for every prime $p$. See for example [3] for a discussion and alternative definitions of the class of FAR groups. In particular all soluble groups of finite (Prüfer) rank are FAR groups. The following summarises our main conclusions in this paper.

THEOREM. Let $\phi$ be an automorphism of the finite extension $G$ of a soluble FAR group. Then the following hold.

i) If $C_{G}(\phi)$ is finite and $\phi^{2}=1$, then $[G, \phi]^{\prime}$ is finite.

ii) If $C_{G}(\phi)$ is finite, then $G /[G, \phi]$ is finite.

iii) If $\phi$ has finite order, then the index $\left(G:[G, \phi] . C_{G}(\phi)\right)$ is finite.

In fact we can weaken the FAR hypothesis in the theorem considerably in many places. For i) it suffices to assume only that $G$ is a group with finite Hirsch number (even in a weak sense). For iii) it suffices to assume that $G$ is a soluble-by-finite group with finite Hirsch number satisfying min- $p$ for all primes $p$ dividing the order of $\phi$. However we do need some min- $p$ hypothesis; suppose $G$ is the direct product of the cyclic groups $\left\langle a_{i j}\right\rangle$ of order $p$ for $i \geq 1, j=1,2$ and $p$ any (fixed) prime. Define $\phi: G \rightarrow G$ by $a_{i 1} \phi=a_{i 1}$ and $a_{i 2} \phi=a_{i 1} a_{i 2}$ for each $i \geq 1$. Then $\phi$ is an automorphism of $G$ of prime order $p$ with $[G, \phi]=C_{G}(\phi)=\left\langle a_{i 1}: i \geq 1\right\rangle$. Thus $\left(G:[G, \phi] . C_{G}(\phi)\right)$ is infinite. Trivially $G$ has Hirsch number 0 . As Endimioni and Moravec point out in [1], we do need $\phi$ of finite order in iii), even if $G$ is free abelian of rank 2. Note that iii) implies that $G /[G, \phi]$ is isomorphic to a finite extension of a section of $C_{G}(\phi)$; see Remark 6 below.

We are unable to weaken the FAR condition in ii) in general, though we can in special cases. Note that ii) implies that $C_{S}(\phi)$ is finite for every $\phi$ invariant section $S$ of $G$. If $\phi$ has finite order (with $C_{G}(\phi)$ finite) it suffices for ii) to hold to assume that $G$ is just soluble-by-finite with finite Hirsch number, see Remark 7. If $\phi$ has prime order, then it suffices simply to assume that $G$ has finite Hirsch number, since Theorem 1 of [6], for example, reduces the problem to the soluble-by-finite and hence previous case. A (torsion-free)-by-finite group with finite Hirsch number is always a finite extension of a soluble FAR group, so this case is also covered. Of course if $G$ satisfies the conclusion of iii) and $C_{G}(\phi)$ is finite, then again $G /[G, \phi]$ is always finite.

If in ii) the order of $\phi$ is infinite, then we do need some sort of min- $p$ condition, even if $\phi$ acts fixed-point freely on $G$. For example, let $G$ 
denote the cartesian product of the additive cyclic groups $\left\langle a_{i}\right\rangle$ of order $p^{i}$, where $p$ is any prime and $i=1,2, \ldots$. Define $\phi: G \rightarrow G$ by $\left(e_{i} \alpha_{i}\right) \phi=$ $\left(\left(e_{i}+p e_{i-1}\right) a_{i}\right)$, where the $e_{i}$ are integers with $e_{0}=0$. Then $\phi$ is easily seen to be an automorphism of $G$ of infinite order with $C_{G}(\phi)=\langle 0\rangle$ and $[G, \phi]=p G$. In particular $G /[G, \phi]$ is infinite and trivially $G$ is abelian with Hirsch number 0 . Clearly $G$ is uncountable, but if $D \leq G$ denotes the direct product of the $\left\langle a_{i}\right\rangle$, then $H=\bigcup_{i>1} D \phi^{-i}$ gives a countable counter example. Finally the converse of ii) is false, even if $G$ is polycyclic and $\phi$ has prime order, see Example 8 below.

By removing from our proof of the above theorem the complications caused by the presence of finite rank but infinitely generated sections, one can use our methods to construct somewhat shorter proofs of i), ii) and iii) than those given in [1]; this is especially the case with iii).

LEMMa 1. Let $G$ be a group with a series of finite length, each factor of which is either periodic or infinite cyclic. If $\phi$ is an automorphism of $G$ with $C_{G}(\phi)$ finite and $\phi^{2}=1$, then $[G, \phi]^{\prime}$ is finite.

Part i) of the Theorem follows at once from this. Note that the condition on $G$ in Lemma 1 is weaker than having finite Hirsch number as we have defined it in the introduction above.

Proof. Now $G$ has $\phi$-invariant normal subgroups $A \leq B$ with $\mathrm{A}$ and G/ $\mathrm{B}$ finite and $\mathrm{B} / \mathrm{A}$ abelian and inverted by $\phi$, see [5] Theorem 2. Also $[G, \phi, B] \leq A$; this follows from [1] Lemma 1 , but it also follows immediately from simply expanding the commutator $[g, \phi, b]$ for any $g \in G$ and $b \in B$. Then $[G, \phi] A / A$ is centre-by-finite. Hence $[G, \phi]^{\prime} A / A$ and $A$ are both finite. Consequently $[G, \phi]^{\prime}$ is finite.

Lemma 2. Let $A$ be an additive torsion-free abelian group of finite rank. If $\phi$ is an automorphism of $A$ with $C_{A}(\phi)=\langle 0\rangle$, then $A / A(\phi-1)$ is finite.

Proof. Clearly $\phi-1$ extends to an automorphism $\theta$ of $V=\boldsymbol{Q} \otimes_{\boldsymbol{Z}} A$. In particular $\operatorname{det} \theta \neq 0$. By the Cayley-Hamilton Theorem $\operatorname{det} \theta \in \boldsymbol{Q}[\theta] \theta \leq$ $\operatorname{End}_{\boldsymbol{Q}} V$. Thus there is a positive integer $e$ with $e \in \boldsymbol{Z}[\theta] \theta=\operatorname{End}_{\boldsymbol{Q}} V$. Hence $e A \leq A . \boldsymbol{Z}[\theta] \theta \leq A \theta=A(\phi-1)$ and therefore $A / A(\phi-1)$ is finite.

For any group $G$ denote the unique maximal locally finite normal subgroup of $G$ by $\tau(G)$. 
LEMMA 3. Let $G$ be a group with finite Hirsch number h and with $\tau(G)$ finite. If $\phi$ is an automorphism of $G$ with $C_{G}(\phi)$ finite, then $G /[G, \phi]$ is finite.

Proof. We induct on $h$; clearly if $h=0$ the claim is vacuous. Suppose $h \geq 1$. Then $G$ has a non-trivial torsion-free abelian characteristic subgroup $A$ with $G / A$ (torsion-free)-by-finite (e.g. see Lemmas 4 and 6 of [6]). Clearly $C_{A}(\phi)=\langle 1\rangle$, so by Lemma 2 we have $(A:[A, \phi])=e$ finite. Set $B=A^{e}$. Then $B$ is characteristic in $G, B \leq[G, \phi]$ and $A / B$ is finite. Further the map $a \mapsto a^{e}$ is an isomorphism of $A$ onto $B$ commuting with $\phi$, so $(B:[B, \phi])=(A:[A, \phi])=e$. By [4] Lemma 1 we have $\left|C_{G / B}(\phi)\right| \leq e\left|C_{G}(\phi)\right|<\infty$. By induction on $h$ the group $G /[G, \phi] B$ is finite. Since $B \leq[G, \phi]$ we have $G /[G, \phi]$ finite.

LEMMA 4. Let $G$ be a finite extension of a soluble FAR group. If $\phi$ is an automorphism of $G$ with $\mid C_{G}(\phi \mid=n$ finite, then $G /[G, \phi]$ is finite.

Part ii) of the Theorem follows at once from Lemma 4.

Proof. Set $\pi=\{$ primes $p: p \leq n\}$ and $T=\tau(G)$. By [2] 3.17 there is a characteristic series

$$
\langle 1\rangle=T_{0} \leq T_{1} \leq \ldots \leq T_{s} \leq T \leq G
$$

of $G$ with each $T_{i} / T_{i-1}$ an abelian $\pi^{\prime}$-group for $i<s, T_{s} / T_{s-1}$ a divisible abelian $\pi$-group and $T / T_{s}$ finite.

Suppose $A=T_{1}$ is a $\pi^{\prime}$-group. Clearly $C_{A}(\phi)=\langle 1\rangle$. Then the map $\gamma: a \mapsto a^{-1} . a \phi$ is an isomorphism of $A$ into itself. If $A_{p}$ is the $p$-primary component of $A$, then $A_{p} \leq A_{p} \gamma$. If $A_{p}>A_{p} \gamma$, then $A_{p} \gamma^{i}>A_{p} \gamma^{i+1}$ for all $i \geq 0$. But $A_{p}$ satisfies the minimal condition ( $G$ satisfies min- $p$ for all primes $p$ recall). Therefore $A_{p}=A_{p} \gamma$ for all $p$ and $A=A \gamma \leq[G, \phi]$. Also if $K / A=C_{G / A}(\phi)$, then Lemma 1 of [4] yields that $(K: A) \leq n$.

Now suppose the $A=T_{1}$ is a divisible abelian $\pi$-group (the case $s=1$ ). Then $A \gamma$ is divisible and hence is a direct summand of $A$. But $\left.\operatorname{ker} \gamma\right|_{A}=C_{A}(\phi)$ is finite so $A \cong A \gamma$ and also $A$ is a direct product of a finite (invariant) number of Prüfer groups. Therefore $A \gamma=A$. Again we obtain $(K: A) \leq n$.

A simple induction on s shows that $T_{s} \leq[G, \phi]$ and that the centralizer of $\phi$ on $G / T_{s}$ has order at most $n$. Further $\tau\left(G / T_{s}\right)$ is finite. Therefore $G /[G, \phi] T_{s}$ is finite by Lemma 3. Consequently $G /[G, \phi]$ is finite. 
LEMMA 5. Let G be a soluble-by-finite group with finite Hirsch number and $\phi$ an automorphism of $G$ of finite order $m$. Suppose $G$ satisfies min- $p$ for every prime $p$ dividing $m$. Then $\left(G:[G, \phi] C_{G}(\phi)\right)$ is finite.

Part iii) of the Theorem follows from Lemma 5.

Proof. If $N$ is a $\phi$-invariant subgroup of $G$ of finite index with $\left(N:[N, \phi] C_{N}(\phi)\right)$ finite, then $\left(G:[G, \phi] C_{G}(\phi)\right)$ is also finite. In particular we may assume that $G$ is soluble. Set $C=C_{G}(\phi)$.

Suppose first that $T=\tau(G)$ is finite. We prove this case by induction on the Hirsch number of $G$. If $G$ is finite the conclusion is vacuous. If not $G$ has a non-trivial torsion-free abelian characteristic subgroup $A$ with $G / A$ (torsion-free)-by-finite ([6] Lemmas 4 and 6 again). Define the map $\gamma: G \rightarrow G$ by $g \mapsto[g, \phi]$ and let $\psi$ denote the endomorphism $1+\left.\phi\right|_{A}+\left(\left.\phi\right|_{A}\right)^{2}+\ldots .+\left(\left.\phi\right|_{A}\right)^{m-1}$ of A. Clearly $\psi\left(\left.\gamma\right|_{A}\right)=0$, so $A \psi \leq C$. Also if $a \in A$, then $a^{m}$ is congruent to $a \psi$ modulo $A \gamma$. Therefore $A^{m} \leq A \gamma \cdot A \psi \leq[A, \phi](A \cap C)$.

Let $A^{m} \leq K \leq G$ with $K / A^{m}$ the centralizer in $G / A^{m}$ of $\phi$. Now $A / A^{m}$ is finite since $A$ has finite rank, so induction applies to $G / A_{m}$. Consequently we may assume that $(G:[G, \phi] K)$ is finite. Also $\left(A \cap K: A^{m}\right)$ is finite and $K=(A \cap K) C$, the latter by Lemma 15 of [6]. Therefore $\left(K: A^{m} C\right)$ is finite; thus $\left(G:[G, \phi] A^{m} C\right)$ is finite. But $A^{m} \leq[A, \phi](A \cap C)$. Consequently $(G:[G, \phi] C)$ is finite. This completes the proof of the $T$ finite case.

Let $\pi$ denote the set of prime divisors of $m$. By [2] 3.17 there is a $\phi$ invariant normal series $\langle 1\rangle=T_{0} \leq T_{1} \leq \ldots \leq T_{s} \leq T$ of $G$ as in the proof of Lemma 4, with factors $T_{i} / T_{i-1}$ abelian $\pi^{\prime}$-groups or divisible abelian $\pi$ groups and $T / T_{s}$ finite. For each $i$ the section $\left\{x \in T_{i}: x^{m} \in T_{i-1}\right\} / T_{i}$ is finite. Hence by our initial remark we may assume that each of these sections is central in $G$. By the $T$-finite case we may assume that $G / T_{s}$ satisfies the conclusion of the lemma. Thus by induction on $\mathrm{s}$ we may assume that $G$ has a periodic abelian characteristic subgroup $A$ with $A^{m}=A, B=\left\{a \in A: a^{m}=1\right\}$ finite and central in $G$ and $(G:[G, \phi] K)$ finite, where $K$ is given by $K / A=C_{G / A}(\phi)$.

Let $k \in K$. Then $k \gamma \in A=A^{m}$, say $k \gamma=a^{m}$ for $a \in A$. Now $a^{m} \in a \psi A \gamma$ and $a \psi \gamma=1, \psi$ being as in the $T$-finite case. Thus $a^{m}=c . d \gamma$ for $c=a \psi \in C$ and some $d \in A$. Hence $k \gamma=c . d \gamma$ and

$$
\left(k d^{-1}\right) \gamma=d . k \gamma \cdot d^{-1} \phi=d . c . d \gamma \cdot d^{-1} \phi=c \in A \cap C .
$$


Note that the argument also implies that $A=A \gamma(A \cap C)=$ $[A, \phi](A \cap C)$. Since the actions of $k$ and $\phi$ on $A$ commute, so $k$ and $k d^{-1}$ normalize $A \cap C$. Then $\phi$ stabilizes the series

$$
\langle 1\rangle \leq A \cap C \leq\left\langle k d^{-1}\right\rangle(A \cap C)
$$

and hence $\left[k d^{-1}, \phi\right]^{m}=\left[k d^{-1}, \phi^{m}\right]=1$. Thus $k d^{-1} \in L$, where $L / B=C_{G / B}(\phi)$. Hence $K \leq L A=A L$; indeed $K=A L$. Also $\phi$ stabilizes the series $\langle 1\rangle \leq B \leq L$ and $B$ is central in $L$. Therefore $\gamma_{L}$ is a homomorphism of $L$ into $B$ with kernal $C$. Thus $(L: C) \leq|B|<\infty$. Consequently $(K: A C)$ is finite and hence $(G:[G, \phi] A C)$ is finite. As we saw above $A=[A, \phi](A \cap C)$. Therefore $(G:[G, \phi] C)=(G:[G, \phi][A, \phi] C$ is finite, as required.

Remark 6. Suppose $G$ and $\phi$ are as in Lemma 5. Then always $G /[G, \phi]$ is a finite extension of some section of $C_{G}(\phi)$. For if $N$ denotes the intersection of the conjugates in $G$ of $[G, \phi] C_{G}(\phi)$, then $G / N$ is finite by Lemma 5 and $[G, \phi] \leq N$. Thus $N=[G, \phi] C_{N}(\phi)$ and $N /[G, \phi] \cong C_{N}(\phi) /\left([G, \phi] \cap C_{N}(\phi)\right)$.

In particular if $\boldsymbol{X}$ is a subgroup and image closed class of groups containing $C_{G}(\phi)$ and if $\boldsymbol{F}$ denotes the class of finite groups, then $G /[G, \phi] \in \boldsymbol{X F}$. If $G$ is polycyclic, this is Proposition 1 of [1].

REMARK 7. Let $G$ be a soluble-by-finite group with finite Hirsch number. If $\phi$ is an automorphism of $G$ of finite order with $C_{G}(\phi)$ finite, then $\mathrm{G} /[\mathrm{G}, \phi]$ is finite.

Proof. Set $T=\tau(G)$. Repeated use of [6] Lemmas 9 and 13a) yield that $T /[T, \phi]$ and $C_{G / T}(\phi)$ are both finite. Then $G /[G, \phi] T$ is finite by Lemma 5 (or Lemma 3 if you prefer). Trivially $[T, \phi] \leq[G, \phi]$. Therefore $G /[G, \phi]$ is finite.

EXAMPLE 8. Let $\omega$ be a primitive $p$-th root of unity in $\boldsymbol{C}$ for some prime $p$ and put $R=\boldsymbol{Z}[\omega] \leq \boldsymbol{C}$ and $G=\operatorname{Tr}_{1}(3, R)$. (Here $\boldsymbol{Z}$ denotes the integers, $\boldsymbol{C}$ the complex numbers and $\operatorname{Tr}_{1}(3, R)$ the lower unitriangular group of degree 3 over the ring $R$; later $\boldsymbol{Q}$ will denote the rational numbers.) Then $G$ is a $2(p-1)$-generator nilpotent group of class 2 (and in particular is polycyclic). If $Z$ denotes the centre of $G$ and $\left\{e_{i j}\right\}$ the standard set of matrix units in $C^{3 \times 3}$, then $Z=1+\operatorname{Re}_{31}=G^{\prime}$ and $Z \cong R$ and $G / Z \cong R \oplus R$.

Let $\phi$ denote the automorphism of $G$ induced by conjugation by the diagonal matrix $\operatorname{diag}(1, \omega, 1)$. Then $\phi$ has order $p$, centralizes $Z$ and acts 
fixed-point freely on $G / Z$. If $x=1+a e_{21}$ and $y=1+b e_{32}$ for some $a$, $b \in R$, then a simple calculation shows that

$$
\left[x^{-1} \cdot x \phi, y^{-1} \cdot y \phi\right]=1+(\omega-1)^{2} \omega^{-1} a b e_{31} \in[G, \phi]^{\prime} .
$$

Also $1+\omega+\omega^{2}+\ldots+\omega^{p-1}=0$, so $p=\sum_{1 \leq i<p}\left(1-\omega^{i}\right) \in(\omega-1) R$. Therefore $1+p^{2} \operatorname{Re}_{31} \leq Z \cap[G, \phi]^{\prime}$ and in particular $([G, \phi] Z:[G, \phi])=$ $(Z: Z \cap[G, \phi])$ divides $p^{2(p-1)}$.

Suppose $g=\left(g_{i j}\right) \in G$. The map $g \mapsto\left(g_{21}, g_{32}\right)$ is a homomorphism of $G$ onto $R \oplus R$ with kernel $Z$ that maps $[G, \phi]$ onto $(\omega-1)(R \oplus R)$. Thus $(G:[G, \phi] Z)$ also divides $p^{2(p-1)}$. Consequently $G /[G, \phi]$ is finite of order dividing $p^{4(p-1)}$. However $C_{G}(\phi)=Z$, which is infinite. Thus the converse of Part ii) of the Theorem does not hold, even for polycyclic groups and automorphisms of finite prime order.

Suppose instead of $\operatorname{Tr}_{1}(3, R)$ we set $G=\operatorname{Tr}_{1}(3, K)$, where $K$ is the field $\boldsymbol{Q}[\omega] \leq \boldsymbol{C}$, but with $\phi$ still given by conjugation by $\operatorname{diag}(1, \omega, 1)$. Then here $G$ is torsion-free nilpotent of finite rank and class 2 and $Z=C_{G}(\phi) \cong K$ is infinite, $Z$ being the centre of $G$ again. Our previous argument shows that $G /[G, \phi]$ has finite exponent (dividing $p^{3}$ ). But $G$ is divisible, so here we have $|\phi|=p, G=[G, \phi]$ and $C_{G}(\phi)$ infinite.

\section{REFERENCES}

[1] G. ENDIMIONI - P. MoRAVEC, On the centralizer and the commutator subgroup of an automorphism, Monatsh. Math., to appear.

[2] O. H. KegEL - B. A. F. Wehrfritz, Locally Finite Groups, North Holland Pub. Co., Amsterdam 1973.

[3] D. J. S. Robinson, Finiteness Conditions and Generalized Soluble Groups (2 vols.), Springer-Verlag, Berlin 1972.

[4] B. A. F. WeHrfritz, Almost fixed-point-free automorphisms of soluble groups, J. Pure \& Appl. Algebra, 215 (2011), pp. 1112-1115.

[5] B. A. F. Wehrfritz, Almost fixed-point-free automorphisms of order 2, Rend. Circ. Mat. Palermo, 60 (2011), pp. 365-370.

[6] B. A. F. WeHrFritz, Almost fixed-point-free automorphisms of prime order, Cent. Eur. J. Math. 9 (2011), pp. 616-626.

Manoscritto pervenuto in redazione il 5 settembre 2011. 